\title{
Low-Bit Quantization Methods for Modulated Wideband Converter Compressed Sensing
}

\author{
Lap Luat Nguyen*, Duy H. N. Nguyen ${ }^{\dagger}$, Anthony Fiche*, Thang Huynh ${ }^{\S}$ and Roland Gautier* \\ *Univ Brest, Lab-STICC, CNRS, UMR 6285, F-29200, Brest, France \\ *Email: \{firstname.name\} @ univ-brest.fr \\ ${ }^{\dagger}$ Department of Electrical and Computer Engineering, San Diego State University, CA, USA \\ ${ }^{\dagger}$ Email: duy.nguyen@sdsu.edu \\ $\S$ Department of Mathematics, University of California San Diego, CA, USA \\ §Email: tlh007@ucsd.edu
}

\begin{abstract}
This paper studies the impacts of low-bit quantization to wideband signal reconstruction with a sub-Nyquist sampling scheme Modulated Wideband Converter (MWC). Several real types of analog-to-digital converters (ADCs) are firstly simulated to the MWC and their results on wideband signal reconstruction are compared based on the low-bit criterion of the ADCs. Assessing the wideband reconstruction performances, this study proposes a trade-off between the oversampling factor, the number of bits and the type of quantizer, which can achieve the performance of reconstruction as close as possible to the ideal reconstruction.
\end{abstract}

Index Terms-Modulated Wideband Converter, spectrum sensing, sub-Nyquist sampling, $\Sigma \Delta$ analog-to-digital converter.

\section{INTRODUCTION}

Sampling a wideband signal is a challenging task due to the problems of large bandwidth requirement and the limited sampling rate of conventional ADCs. In the context of spectrum sensing where the carrier frequency is unknown, the whole bandwidth of sensing signal may need to be digitally converted. As a result, the high sampling rate may exceed the capabilities of ADC hardware. If the bandwidth of each sensed signal rises up to several tens of $\mathrm{GHz}$, the NyquistShannon theorem states that the sampling rate should be at least twice the signal bandwidth [1], at beyond the capability of any traditional ADCs. Consequently, some methods to reduce the sampling rate have been presented in literature. Especially, the Modulated Wideband Converter (MWC) scheme [2] relying on Compressed Sensing (CS) theory [3] [4] is under the hypothesis that the signal to be sensed is sparsely distributed across the wideband spectrum.

MWC is attractive for practical implementation since it solves the problem of the high sampling rate requirement by reducing the input bandwidth of wideband signal and this bandwidth can be sampled by a conventional ADC at a normal rate, usually called sub-Nyqusit sampling system. The MWC consists of a multi-branch of analog devices, each branch includes a mixer, a lowpass filter and an ADC. Besides, the MWC must meet two requirements so that the wideband signal can be reconstructed accurately. First, the wideband input signal must comprise of sparse sub-channels. Secondly, all analog devices in the MWC scheme are assumed to be ideal [5]. For example, the MWC output can be reconstructed when it is obtained from an ideal lowpass filter (flat passband, nonexistence phase shift, transition band and stopband) and high resolution ADCs without any distortion. In [6], it has been demonstrated that MWC can be implemented with non-ideal hardware (mixers, lowpass filters) with proposed calibration method to the analog and physical distortion or imperfections. In addition, to implement the analog compressed acquisition chain of the MWC, the impacts of each non-ideal component need to be evaluated independently. By assuming the other components ideally, in [7], it has been demonstrated that MWC can be implemented with non-ideal and commercial off-theshelf SXLP-36+ lowpass filter with little performance loss. This paper continually focuses on the implementation MWC system with non-ideal low-bit ADCs.

The benefits of low-bit ADCs are low cost, low power consumption in case of green communications and simple hardware. Furthermore, the low-bit ADCs have not been thoroughly investigated in the context of MWC. The main constraint of a low-bit ADC, however, is the significant quantization error between input and quantized output. The advantage of a lowbit memoryless ADC is that it can be deployed in the MWC easily, and a common way to reduce the quantization error is to implement oversampling [8]. Nevertheless, in the context of wideband sampling, the MWC scheme aims to reduce sampling rate into sub-Nyqusit rate. Thus, we take an alternative approach by increasing the sub-Nyquist sampling rate by only a few times. In addition, 1-bit $\Sigma \Delta \mathrm{ADC}$ is studied in the MWC due to its noise shaping capability of the quantization error at baseband. We then show that the $\Sigma \Delta$ ADC can reduce the quantization error better than a memoryless ADC applied with an optimal quantization technique at moderate oversampling values. The main motivation of this paper is to reproduce a prototype of a CS scheme based on the MWC with non-ideal low-bit ADCs for spectrum monitoring applications (cognitive network, cellular network, Internet-of-Things...). Issued from our simulation results, a trade-off is proposed between oversampling rate and number of bits of an ADC which can be reasonable for a sub-Nyquist sampling system under the condition of low-bit, low consumption and hardware complexity.

The paper is organized as follows: Section II presents back- 
ground and theory of the MWC. In Section III, the lowbit memoryless ADC is introduced and simulated followed by a presentation of the oversampling process. Section IV explains the $\Sigma \Delta$ ADC and section $\mathrm{V}$ presents the performances of reconstruction and theoretical Signal-to-Quantization-Noise Ratio $\left(\mathrm{SNR}_{Q}\right)$ between different low-bit ADCs. The conclusion is given in Section VI.

\section{Modulated Wideband CONVERTER}

The sub-Nyquist sampling MWC scheme is illustrated in Figure 1. On each channel in time domain, the input signal $x(t)$ is firstly split into $M$ replicas, corresponding to $M$ channels of the MWC. Then each replica is multiplied by a periodic mixing function $p_{i}(t)$, with $1 \leq i \leq M$. The result $x_{i}(t)$ is continually filtered and converted to digital signal $y_{i}[n]$.

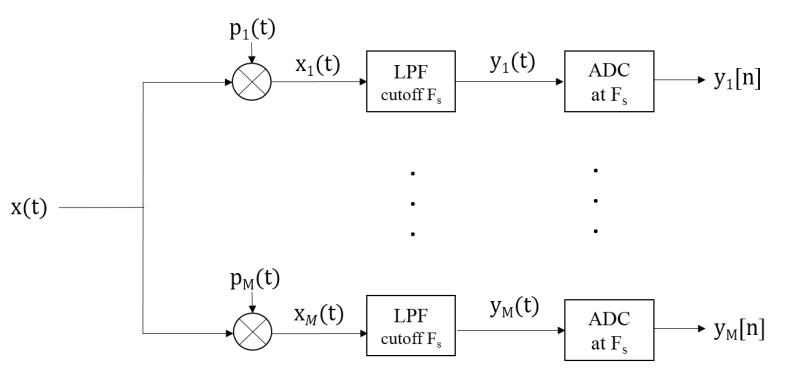

Fig. 1: The block diagram of the Modulated Wideband Converter (MWC).

The principle of the sub-Nyquist sampling MWC scheme is to shift all the subbands of the wideband input signal into baseband. This can help reduce the sampling rate since all useful information of the input signal at baseband will be kept by the lowpass filter. To perform this assumption, the input signal must follow the condition of sparsity [5], [9].

Assuming that the mixing function $p_{i}(t)$ is periodic with $T_{p}=1 / F_{p}$. On each channel of the MWC, the input signal is multiplied by a $T_{p}$ periodic sequence. Then, sampling rate of the ADC is denoted $F_{s}=1 / T_{s}$ and for convenience, the cutoff frequency of the lowpass filter is chosen in $\left[\frac{-F_{s}}{2} ; \frac{F_{s}}{2}\right]$. Hence, the system in frequency domain is:

$$
X_{i}(f)=\left(P_{i} * X\right)(f)
$$

where $P_{i}(f), X(f)$ are Fourier transform of mixing function $p_{i}(t)$ and input signal $x(t)$, respectively. After the lowpass filter, the filtered signal $Y_{i}(f)$ has the spectrum:

$$
Y_{i}(f)=H(f) X_{i}(f)
$$

where $H(f)$ is the lowpass filter transfer function. Lastly, the MWC output at $F_{s}$ sampling rate is:

$$
\tilde{Y}_{i}(f)=F_{s} \sum_{k=-\infty}^{+\infty} Y_{i}\left(f-k F_{s}\right)
$$

where $\tilde{Y}_{i}(f)$ denotes the spectrum of the sampled signal $y_{i}[n]$ in frequency domain. The system equation can be rewritten by combining equations (1), (2) and (3):

$$
\tilde{Y}_{i}(f)=F_{s} \sum_{k=-\infty}^{+\infty} H\left(f-k F_{s}\right)\left(P_{i} * X_{i}\right)\left(f-k F_{s}\right) .
$$

Therefore, with $P_{i}(f)=\sum_{l=-\infty}^{+\infty} p_{i l} \delta\left(f-l F_{p}\right), p_{i l}=$ $F_{p} \int_{-T_{p} / 2}^{T_{p} / 2} p_{i}(t) e^{-j 2 \pi l F_{p} t} d t$ is simply the Fourier coefficient decomposed from $p_{i}(t)$. Equation (4) can be derived as:

$$
\tilde{Y}_{i}(f)=\sum_{l=-\infty}^{+\infty} p_{i l} F_{s} \sum_{k=-\infty}^{+\infty} H\left(f-k F_{s}\right) X\left(f-l F_{p}-k F_{s}\right)
$$

Let us denote $Z_{l}(f)=H(f) X\left(f-l F_{p}\right)$ as the signal $X(f)$ shifted by $l F_{p}$, and $Z_{l}(f)$ keeps only a bandwidth $F_{s}$ at baseband after lowpass filter $H(f)$. Equation (5) then becomes:

$$
\begin{aligned}
\tilde{Y}_{i}(f) & =\sum_{l=-\infty}^{+\infty} p_{i l} F_{s} \sum_{k=-\infty}^{+\infty} Z_{l}\left(f-k F_{s}\right) \\
& =\sum_{l=-\infty}^{+\infty} p_{i l} \tilde{Z}_{l}(f) .
\end{aligned}
$$

The expression $F_{s} \sum_{k=-\infty}^{+\infty} Z_{l}\left(f-k F_{s}\right)$ is the Fourier transform of sampled signal $z_{l}[n]$ at sampling frequency $F_{s}$. The mixing sequence is periodic, thus, the summation from infinity is then bounded in $\left[-L_{0}, L_{0}\right]$ with $-L_{0} \leq l \leq L_{0}$. The system equation (6) in time domain is $y_{i}[n]=\sum_{l=-L_{0}}^{\bar{L}_{0}} p_{i l} z_{l}[n]$. In [9], $L$ is defined as a ratio between the $F_{n y q}$ and $F_{p}$, such as $L=F_{n y q} / F_{p}$ and $L_{0}=\frac{L-1}{2}$. Hence, $L$ is the size of the mixing sequences. The notations $y_{i}[n]$ and $z_{l}[n]$ are inverse Fourier transform of $\tilde{Y}_{i}(f)$ and $Z_{l}(f)$ respectively. Finally, the whole system can be simplified in matrix form as:

$$
\mathbf{y}=\mathbf{P z},
$$

with $(\mathbf{P})_{i l}=p_{i l}$ is a $M \times L$ matrix, $(\mathbf{y})_{i}=y_{i}[n]$ is a $M \times 1$ vector and $(\mathbf{z})_{l}=z_{l}[n]$ is a $L \times 1$ vector. From these assumptions, the equivalent model to the MWC [5], [9] is shown in Figure 2.

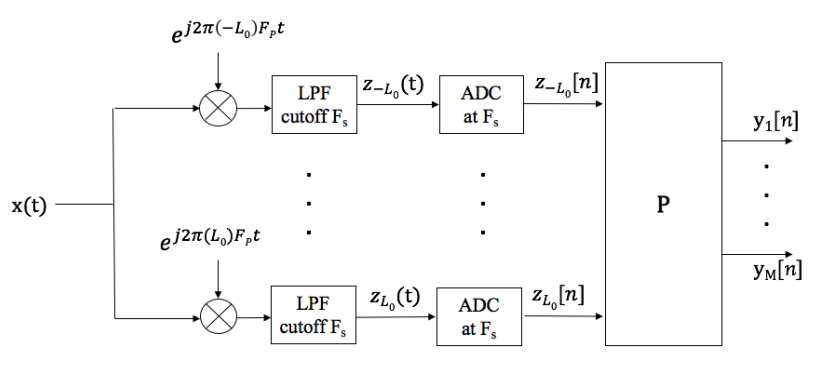

Fig. 2: Equivalent scheme of MWC.

In practice, the series of mixers, lowpass filters and ADCs deployed for all $M$ channels are high-cost, which makes the MWC scheme to be impractical for high $M$. Hence, by introducing a collapsing factor $q=F_{s} / F_{p}$ [9], this expression 
can be understood as dividing the input signal sampled at $F_{s}$ into $q$ signals sampled at $F_{p}$. Theoretically, the output of the MWC can be considered as sampled at $F_{p}$ with $q \times M$ channels at the input. The following section introduces the uniform memoryless low-bit ADCs with an optimal quantizing method and the oversampling technique. These low-bit ADCs are deployed in the MWC by simulation. The performances of correct reconstruction and false alarm are then evaluated.

\section{LOW-BIT MEMORYLESS ADC AND OVERSAMPLING METHOD}

\section{A. Low-bit memoryless ADC}

The signal after lowpass filtering of the MWC is then passed through an ADC. There are two steps in this stage, sampling and quantizing. The sampling process is to convert the analog signal to discrete samples and the quantizing process is to map these samples into the predetermined voltage levels of the device, which correspond to the output digital codes of the ADC. Increasing number of bits in ADC is to reduce the quantization error but it leads to high-cost and more complex hardware. Thus, this paper focuses on low-bit quantizer and studies an optimal quantizing technique which can achieve high performance on the MWC system.

The easiest way to quantize an analog signal is peak-topeak quantization. However, an optimal quantization scheme must take into account input signal distribution [10]. Let us assume that the signal after lowpass filter of the MWC has Gaussian distribution, the optimal Gaussian source quantizing technique [11] is applied for a common mid-rise quantizer. The impact of this real ADC into outputs of the MWC system is evaluated based on performances of correct reconstruction rate $P_{c}=\frac{\%\left(B_{r} \cap B_{d}\right)}{\% B_{r}}$ and false alarm rate $P_{f}=\frac{\%\left(\left(B_{d} \backslash B_{r}\right) \cap \bar{B}_{r}\right)}{1-\% B_{r}}$, with $B_{r}$ the real subbands from input signal and $B_{d}$ the detected subbands. The method on estimating these performances was explained in [7].
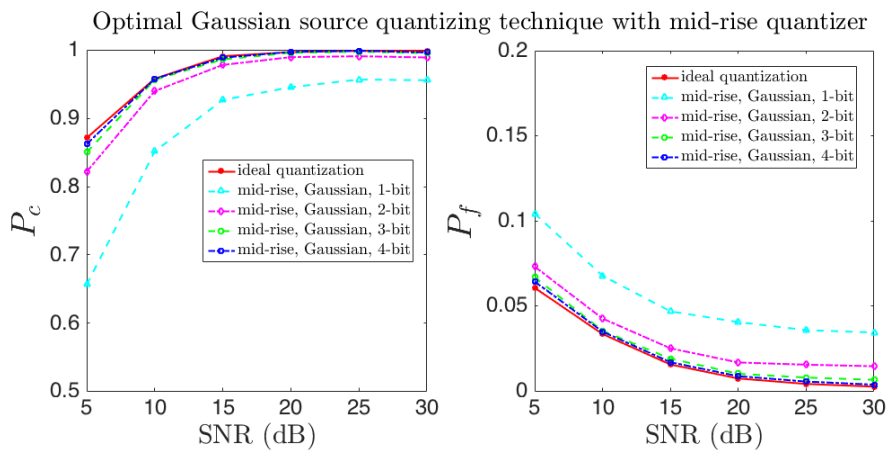

Fig. 3: Correct reconstruction and false alarm rates in function of SNRs (dB) obtained by the MWC with 1 to 4 bits common mid-rise quantizers applying optimal Gaussian source quantizing technique.

The memoryless 1 to 4-bit mid-rise ADCs applying an optimal Gaussian source quantizing technique are firstly simulated in the MWC scheme (in Figure 1) with assumptions that the mixers and the lowpass filters are ideal. The performances of these real ADCs based on correct reconstruction and false alarm are compared to the ideal ADC as in Figure 3. The performances of correct reconstruction and false alarm are in function of SNR changing from 0 to $30 \mathrm{~dB}$. In the simulation, the parameters of the MWC are chosen as the number of MWC channels $M=4$, the collapsing factor $q=7$, the ratio between mixing frequency and Nyquist frequency $L=96$, and the Nyquist frequency $F_{n y q}=1 \mathrm{GHz}$. Assuming that in $\left[0, F_{n y q} / 2\right]$, there are six subbands which can be reconstructed $N_{t}=6$. The bandwidth of each subband is $B=7 \mathrm{MHz}$ with identical power levels.

It can be observed in Figure 3 that high-bit ADCs need to be deployed (3 and 4-bit) to approach the ideal quantization. The main problem of low-bit quantization is the quantization error. To reduce the quantization error, the oversampling technique will be presented to the MWC in the following section.

\section{B. Oversampling}

The quantization error is produced during the quantized process, and usually called quantization noise. The quantized sample is given by:

$$
\hat{y}_{i}[n]=y_{i}[n]+e_{i}[n]
$$

with $e_{i}$ is the quantization noise. Indeed, when $\hat{y}_{i}$ is chosen at mid-point of quantization regions, the random additive noise $e_{i}$ has amplitude distribution in $-\Delta / 2 \leq e_{i} \leq \Delta / 2$ (with $\Delta$ is quantization interval). In the MWC system, the input signal is filtered at $F_{c}=F_{s} / 2$ and then sampled at $F_{s}$ with the root mean square of quantization noise is $E(f)=\frac{\Delta}{\sqrt{12}}$ [12]. Thus, the distribution of quantization noise is uniform in $F_{s}$ bandwidth. The quantization noise spectral density in $F_{s}$ bandwidth is:

$$
N_{e}(f)=\frac{\left|E(f)^{2}\right|}{F_{s}}=\frac{\Delta^{2}}{12 F_{s}} .
$$

At this time, the MWC filtered signal is bounded in $\left[-F_{c}, F_{c}\right]$ at baseband. Finally, this baseband quantization noise spectral density is:

$$
S_{B}=\int_{-F_{c}}^{F_{c}} N_{e}(f) d f=\frac{\Delta^{2}}{12}\left(\frac{2 F_{c}}{F_{s}}\right)=\frac{\Delta^{2}}{12} .
$$

Let us denote $m$ is the oversampling factor with $F_{s}^{\prime}=m F_{s}$, when $F_{s}^{\prime}$ is a sampling frequency greater than $F_{s}, m$ times. Hence,

$$
S_{B m}=\frac{\Delta^{2}}{12}\left(\frac{2 F_{c}}{F_{s}^{\prime}}\right)=\frac{\Delta^{2}}{12}\left(\frac{2 F_{c}}{m F_{s}}\right)=\frac{S_{B}}{m} .
$$

From (11), it can be seen that oversampling helps reducing the noise power spectral density. Thus, the lowpass filter and downsampling process would reduce the filtered noise power. The hypothesis of the MWC system is to sample under the Nyquist rate, $F_{s} \ll F_{n y q}$. Hence, the moderate value of $m$ needs to be chosen carefully to meet this condition of subNyquist sampling, $m F_{s} \ll F_{n y q}$. Figure 4 shows a conventional MWC system and its oversampling counterpart. The decimator in oversampling scheme plays a role as a digital filter, to suppress the samples outside filtered bandwidth $F_{c}$. 
Overall, the memoryless quantizer is easy to implement into the MWC and it can improve $3 \mathrm{~dB}$ in Signal-to-QuantizationNoise Ratio $\left(\mathrm{SNR}_{Q}\right)$ per doubling sampling rate [8]. Nevertheless, due to the hypothesis of sub-Nyquist sampling rate, the following section presents the $\Sigma \Delta$ ADC which can shape quantization noise at baseband significantly lower than a memoryless ADC (such as mid-rise ADC) with a moderate oversampling value.

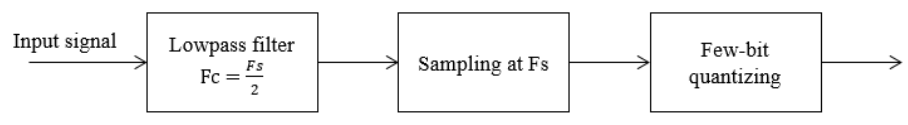

(a) MWC conventional sampling scheme.

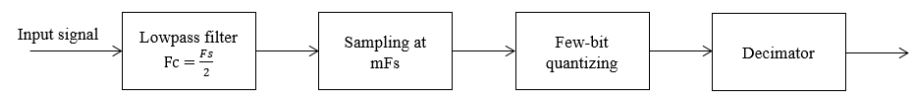

(b) MWC oversampling scheme.

Fig. 4: MWC conventional sampling and oversampling schemes.

\section{IV. $\Sigma \Delta$ ANALOG-TO-Digital CONVERTER}

The 1-bit $\Sigma \Delta$ ADC is a low power consumption and simple hardware [13]. The $\Sigma \Delta$ ADC is a combination of noise shaping, oversampling and decimation stages as shown in Figure 5. The functions of noise shaping and oversampling are performed by the $\Sigma \Delta$ modulator. The first order of $\Sigma \Delta$ modulator is illustrated in Figure 6. The 1-bit digital output of the $\Sigma \Delta$ modulator is then passed through the digital decimation filter. The output of decimation filter provides a digital representation of the quantized input signal at sampling rate $F_{s}$.

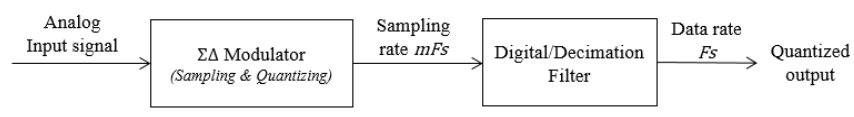

Fig. 5: $\Sigma \Delta$ ADC block diagram.

The $\Sigma \Delta$ modulator includes an analog difference node, an integrator, a 1-bit quantizer and a 1-bit digital-to-analog converter (DAC) in the feedback loop. The signal which is applied to the integrator is the difference between the analog input $y_{i}$ and the predicted analog input from the quantized output $\hat{y}_{i}$ by the DAC. This difference is considered as the quantization error. This quantization error is accumulated by the integrator and the quantized by a 1-bit quantizer. The quantization error of 1-bit quantizer is large, however, the decimation filter can provide an accurate output by averaging the sampled input over several sample periods [14].

In $z$-domain, let us denote the transfer function of the integrator which is $I(z)$, the $\Sigma \Delta$ modulator equation in $z$ domain gives [14]:

$$
\begin{aligned}
\tilde{Y}_{i}(z) & =E_{i}(z)+I(z)\left(Y_{i}(z)-z^{-1} \tilde{Y}_{i}(z)\right) \\
& =E_{i}(z) \frac{1}{1+I(z) z^{-1}}+Y_{i}(z) \frac{I(z)}{1+I(z) z^{-1}},
\end{aligned}
$$

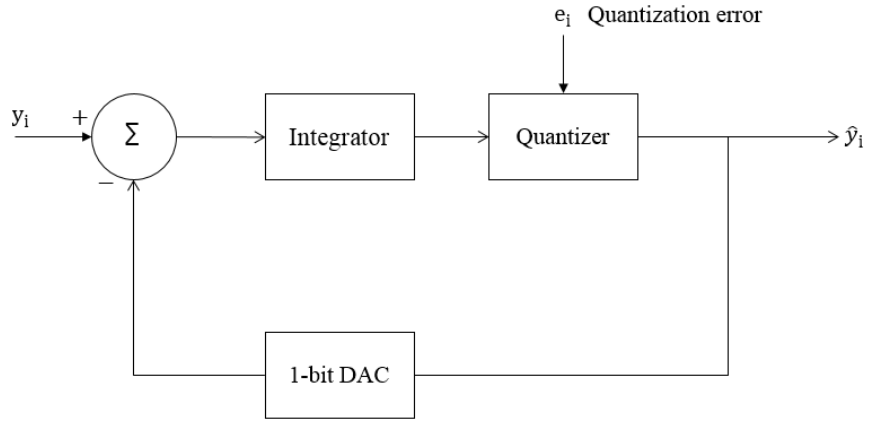

Fig. 6: First-order $\Sigma \Delta$ modulator.

the ideal integrator is considered as $I(z)=\frac{1}{1-z^{1}}$, the output of $\Sigma \Delta$ modulator in (12) can be simplified:

$$
\tilde{Y}_{i}(z)=Y(z)+\left(1-z^{-1}\right) E(z) .
$$

In time domain, the output of the $\Sigma \Delta$ modulator is $\hat{y}_{i \Sigma \Delta}[n]=$ $y_{i}[n]+e_{i}[n]-e_{i}[n-1]$ due to the integrator (accumulating quantization error from the last stage). Let us denote that $n_{e \Sigma \Delta}[n]=e_{i}[n]-e_{i}[n-1]$ is the noise at output of $\Sigma \Delta$ modulator. Thus, in frequency domain $n_{e \Sigma \Delta}$ becomes:

$$
\begin{aligned}
N_{e \Sigma \Delta}(f) & =E(f)-E(f) e^{-j \omega T_{s}^{\prime}} \\
& =2 E(f) \sin \left(\frac{\omega T_{s}^{\prime}}{2}\right) e^{-j\left(\omega T_{s}^{\prime}-\pi\right) / 2},
\end{aligned}
$$

where $E(f)$ is $e_{i}[n]$ in frequency domain and $F_{s}^{\prime}=1 / T_{s}^{\prime}=$ $m F_{s}$ is oversampling frequency of the MWC. Hence, the noise spectral density in $F_{s}^{\prime}$ bandwidth (oversampling of $F_{s}$ ) is $N_{e \Sigma \Delta}=\frac{\left|N_{e \Sigma \Delta}(f)\right|^{2}}{F_{s}^{\prime}}$. Assuming that the $\Sigma \Delta$ has the same quantization level $\Delta$ as mid-rise quantizer in previous section, $E(f)=\Delta / \sqrt{12}$ from equation (9), then $N_{e \Sigma \Delta}=$ $\frac{4 \Delta^{2}\left(\sin \left(\frac{\pi f}{F_{s}^{\prime}}\right)\right)^{2}}{12 F_{s}^{\prime}}$. The baseband quantization noise spectral density is:

$$
S_{B \Sigma \Delta}=\int_{-F_{c}}^{F_{c}} N_{e \Sigma \Delta}(f) d f \approx \frac{\pi^{2}}{3} \frac{S_{B}}{m^{3}},
$$

with $S_{B}$ is the baseband quantization noise spectral density with normal sampling rate $F_{s}$ as in equation (10). It is easy to see from equation (15) that the quantization noise in $\Sigma \Delta$ quantizer is reduced much more than these types of memoryless quantizers as in equation (5) when $m$ is high. Indeed, [8] has shown that by doubling the sampling frequency $F_{s}$, the quantization noise will decrease by $3\left(2 m^{\prime}+1\right) \mathrm{dB}$ in band of interest, with $m^{\prime}$ is doubling factor.

\section{Simulation Results}

In the MWC, the output of lowpass filter is firstly sampled and then quantized. Then, the $\mathrm{SNR}_{Q}$ is computed by comparing the power of the quantization input to the power of the quantization noise:

$$
\mathrm{SNR}_{Q}=10 \log _{10}\left(\frac{\tilde{Y}_{i}^{2}(f)}{E^{2}(f)}\right),
$$


where $E^{2}(f)=\frac{\Delta^{2}}{12}$ and $\Delta=\frac{X_{m}}{2^{n}}$ with $X_{m}$ is the quantization range and $n$ is number of bits for a memoryless uniform quantizer. Hence, equation (16) becomes:

$$
\begin{aligned}
\operatorname{SNR}_{Q} & =10 \log _{10}\left(\frac{12 \tilde{Y}_{i}^{2}(f)}{\frac{X_{m}^{2}}{2^{2 n}}}\right) \\
& =n 20 \log _{10} 2+10 \log _{10} 12+20 \log _{10}\left(\frac{\tilde{Y}_{i}(f)}{X_{m}}\right) \\
& \approx n 6.02+10.79+20 \log _{10}\left(\frac{\tilde{Y}_{i}(f)}{X_{m}}\right)(\mathrm{dB}) .
\end{aligned}
$$

It is easy to see that for the peak-to-peak uniform quantizer, increasing 1 bit can improve $6 \mathrm{~dB}$ in $\mathrm{SNR}_{Q}$. Then, for $m$ times oversampling, the $\mathrm{SNR}_{Q m}$ can improve $10 \log _{10} m(\mathrm{~dB})$. In case of $\Sigma \Delta$ quantizer, the $\mathrm{SNR}_{Q \Sigma \Delta}$ will be:

$$
\begin{aligned}
\operatorname{SNR}_{Q \Sigma \Delta} & =10 \log _{10}\left(\frac{\tilde{Y}_{i}^{2}(f)}{\frac{\Delta^{2}}{12 m^{3}} \times \frac{\pi^{2}}{3}}\right) \\
& =30 \log _{10} m+n 20 \log _{10} 2+10 \log _{10} 12 \\
& +10 \log _{10} 3-20 \log _{10} \pi+20 \log _{10}\left(\frac{\tilde{Y}_{i}(f)}{X_{m}}\right)(\mathrm{dB}) .
\end{aligned}
$$

Hence, when $m=2$, the $\Sigma \Delta$ quantizer can improve $30 \log _{10} m=9 \mathrm{~dB}$ while the memoryless peak-to-peak quantizer can improve only $10 \log _{10} m=3 \mathrm{~dB}$ in the $\mathrm{SNR}_{Q}$. In case of optimal quantizing technique for Gaussian source, let us assume that 1-bit quantizer is used, the quantization level will be 1.59 [11], then the quantization range is $X_{m}=2 \times 1.59=$ 3.18 . Consequently, the quantization noise spectrum will be $E_{G}^{2}(f)=\frac{\Delta^{2}}{12}=\frac{3.18^{2}}{12 \times 2 n}$. Hence:

$$
\begin{aligned}
\operatorname{SNR}_{Q G} & =10 \log _{10}\left(\frac{\tilde{Y}_{i}^{2}(f)}{\frac{3.18^{2}}{12 m \times 2^{2 n}}}\right) \\
& =10 \log _{10} 12-20 \log _{10} 3.18+10 \log _{10} m \\
& +n 20 \log _{10} 2+20 \log _{10}\left(\tilde{Y}_{i}(f)\right)(\mathrm{dB}) .
\end{aligned}
$$

In practice, the quantization range in $\Sigma \Delta$ quantizer is usually set at $X_{m}=4$, it corresponds to the input voltage of the device $2 \mathrm{~V}$. At oversampling factor $m=2$, number of bits $n=1$, the $\operatorname{SNR}_{Q \Sigma \Delta}=8.6+20 \log _{10}\left(\tilde{Y}_{i}\right)(\mathrm{dB})$ while $\mathrm{SNR}_{Q G}=9.8+20 \log _{10}\left(\tilde{Y}_{i}\right)(\mathrm{dB})$. It can be seen that at double sampling rate $(m=2)$, the optimal Gaussian quantizing technique can improve the $\mathrm{SNR}_{Q}$ better than the $\Sigma \Delta$ quantizer. In case $m=4$, however, the $\Sigma \Delta$ quantizer can perform better than the optimal Gaussian quantizer, since $\operatorname{SNR}_{Q \Sigma \Delta}=$ $17.7+20 \log _{10}\left(\tilde{Y}_{i}\right)(\mathrm{dB})$ and $\mathrm{SNR}_{Q G}=12.8+20 \log _{10}\left(\tilde{Y}_{i}\right)$ (dB) at $m=4$ and $n=1$. Figure 7 shows the performances of correct reconstruction and false alarm with the same MWC parameters as in Figure 3.

Figure 7 verified that the improvement in $\mathrm{SNR}_{Q}$ of $\Sigma \Delta$ and Gaussian source mid-rise quantizers are proved by calculating
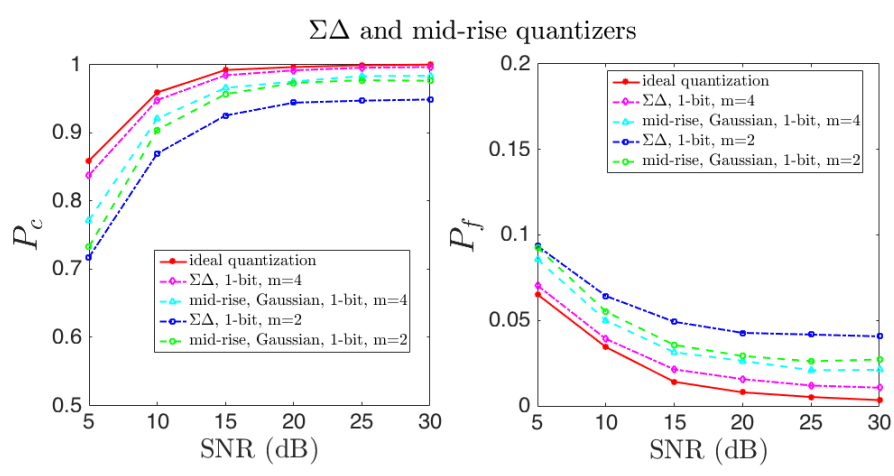

Fig. 7: Correct reconstruction and false alarm rates in function of SNRs $(\mathrm{dB})$ obtained by the MWC with oversampling factor 2 to 4 , 1-bit $\Sigma \Delta$ and Gaussian source mid-rise quantizers.

in equation (18) and (19). It should be noted that the SNR in these figures is different to $\mathrm{SNR}_{Q}$ since SNR is the ratio between wideband input signal and the noise in the communications environment while $\mathrm{SNR}_{Q}$ is the ratio between filtered signal and the quantization noise, which is produced during the quantization process. Moreover, Figure 8 shows an example of spectrum reconstructions at $20 \mathrm{~dB}$ SNR with the ideal $\mathrm{ADC}$ and 1-bit $\Sigma \Delta \mathrm{ADC}$ versus 1-bit Gaussian source mid-rise $\mathrm{ADC}$ at oversampling factors changing from 2 to 4 . It can be observed that at $m=2$, although the locations of input spectrum are reconstructed correctly, there are more reconstructed errors in spectrum reconstructions than at $m=4$.
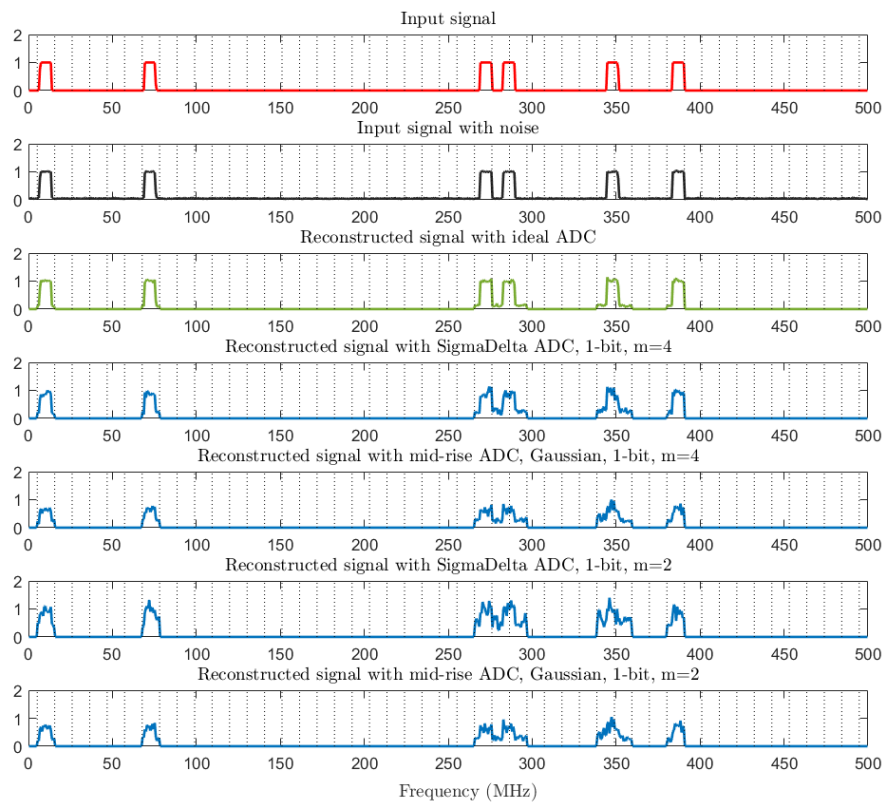

Fig. 8: Example of reconstructed spectra (at $20 \mathrm{~dB}$ SNR) with ideal $\mathrm{ADC}$ and 1-bit $\Sigma \Delta$ ADC versus 1-bit Gaussian source mid-rise ADC at oversampling $m=2$ and $m=4$.

In case 2-bit quantizers, Figure 9 illustrates the performances obtained by the MWC with 2-bit $\Sigma \Delta$ and Gaussian source midrise quantizers and oversampling factors are changed from 2 to 

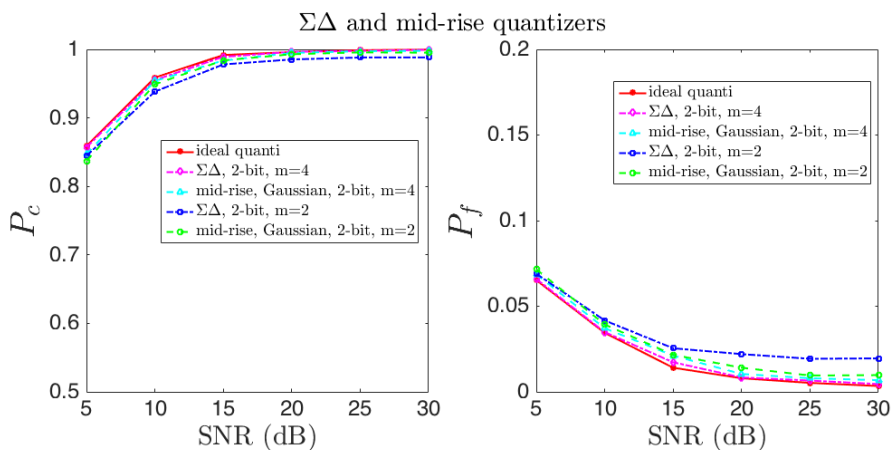

Fig. 9: Correct reconstruction and false alarm rates in function of SNRs (dB) obtained by the MWC with oversampling factor 2 to 4 , 2-bit $\Sigma \Delta$ and Gaussian source mid-rise quantizers.

4.

From the Figures 7 and 9, with oversampling factor $m=2$, the Gaussian source quantizer can perform better than the $\Sigma \Delta$ quantizer even in cases 1-bit and 2-bit. At $m=4$, however, the $\Sigma \Delta$ quantizer can have better performance because equation (15) has shown that the bigger of $m$, the better performance can be archived in $\Sigma \Delta$ quantizer. The simulations in Figures 7 and 9 have verified the theoretical calculation of the $\mathrm{SNR}_{Q}$ above. From these results, they will lead to the trade-off that at 1-bit quantizers, the $\Sigma \Delta$ quantizer with oversampling factor $m=4$ can approach the performance of the ideal quantizer as in Figure 7, because with four times oversampling, the sampling rate $F_{s}^{\prime}$ is still under the Nyquist sampling rate $F_{n y q}\left(F_{s}^{\prime}=\right.$ $\left.4 F_{s} \ll F_{n y q}\right)$, while increasing one bit, the complexity and power consumption of the hardware will increase also [13].

Overall, the simulations in previous section show that a 4bit common mid-rise ADC applying optimal Gaussian source quantizing technique (mid-rise, Gaussian, $n=4, m=1$ ) can provide the performance as well as the ideal ADC. Alternatively, 1-bit $\Sigma \Delta \mathrm{ADC}$ at four times oversampling ( $\Sigma \Delta$, $n=1, m=4$ ) can perform nearly the same the ideal ADC performance. While at the same oversampling factor, a common ADC applying optimal Gaussian source quantizing technique needs 2 bits to approach the same ideal ADC performance (midrise, Gaussian, $n=2, m=4$ ).

\section{CONCLUSION}

To conclude, this subject is attractive in green communications because low-bit ADCs into the MWC under the hypothesis of CS have low power consumption compared to traditional ADCs (16-bit resolution [5]). By the simulation results, a lowbit non-ideal ADC is studied and implemented to the MWC system which can provide an output as close as the ideal one. Then, a trade-off between oversampling factor, number of bits and type of quantizers has been proposed $(\Sigma \Delta: n=1, m=4$ or mid-rise + Gaussian: $n=2, m=2$ ). In the future, a real ADC which exists in the market will be integrated and evaluated. Besides, the power of each subband will be changed to examine the impacts of multi-spectrum input signal and real $\mathrm{ADC}$ in spectrum reconstruction rates in the context of wide spectrum sensing.

\section{REFERENCES}

[1] H. Nyquist, "Certain topics in telegraph transmission theory," Transactions of the American Institute of Electrical Engineers, vol. 47, no. 2, pp. 617-644, 1928.

[2] M. Mishali and Y. C. Eldar, "From theory to practice: Sub-nyquist sampling of sparse wideband analog signals," IEEE J. Sel. Topics in Signal Processing, vol. 4, no. 2, pp. 375-391, 2010.

[3] E. J. Candès, J. Romberg, and T. Tao, "Robust uncertainty principles: Exact signal reconstruction from highly incomplete frequency information," IEEE Trans. Inform. Theory, vol. 52, no. 2, pp. 489-509, 2006.

[4] D. L. Donoho, "Compressed sensing," IEEE Trans. Inform. Theory, vol. 52, no. 4, pp. 1289-1306, 2006.

[5] M. Mishali, Y. C. Eldar, O. Dounaevsky, and E. Shoshan, "Xampling: Analog to digital at sub-Nyquist rates," IET Circuits, Devices \& Systems, vol. 5, no. 1, pp. 8-20, 2011.

[6] D. Cohen, S. Tsiper, and Y. C. Eldar, "Analog-to-digital cognitive radio: Sampling, detection, and hardware," IEEE Signal Processing Magazine, vol. 35 , no. 1 , pp. $137-166,2018$.

[7] L. L. Nguyen, A. Fiche, R. Gautier, C. Canaff, E. Radoi, and G. Burel, "Implementation of Modulated Wideband Converter Compressed Sensing scheme based on COTS lowpass filter with amplitude and phase compensation for spectrum monitoring," in Proc. of the 15th IEEE International Conf. on Advanced Video and Signal Based Surveillance, pp. 1-6, Auckland, New Zealand, 2018.

[8] J. C. Candy, "Oversampling methods for A/D and D/A conversion," Oversampling Delta-Sigma Data converters, 1991.

[9] M. Mishali, Y. C. Eldar, and A. J. Elron, "Xampling: Signal acquisition and processing in union of subspaces," IEEE Trans. Signal Processing, vol. 59, no. 10, pp. 4719-4734, 2011.

[10] J. G. Proakis, M. Salehi, N. Zhou, and X. Li, Communication systems engineering, vol. 2. Prentice Hall New Jersey, 1994.

[11] J. Max, "Quantizing for minimum distortion," IRE Trans. Inform. Theory, vol. 6 , no. 1 , pp. 7-12, 1960.

[12] W. R. Bennett, "Spectra of quantized signals," The Bell System Technical Journal, vol. 27, no. 3, pp. 446-472, 1948.

[13] M. José, "Sigma-Delta Modulators: Tutorial overview, design guide, and state-of-the-art survey," IEEE Trans. Circuits and Systems I: Regular Papers, vol. 58, no. 1, pp. 1-21, 2011.

[14] S. Park, "Principles of Sigma-Delta modulation for Analog-to-Digital Converters," 1999. 